\title{
Effect of Intramolecular Crosslinks on Microgel and Macrogel Formation by Photocrosslinking Reaction in Solution
}

\author{
Yoichi SHIndo, Tokuko Sugimura, Kazuyuki Horie,* \\ and Itaru MITA* \\ Department of Chemistry, Faculty of Science, Toho University, \\ 2-2-1, Miyama, Funabashi-shi 274, Japan \\ * Research Center for Advanced Science and Technology, \\ The University of Tokyo, 4-6-1, Komaba, \\ Meguro-ku, Tokyo 153, Japan
}

(Received June 21, 1989)

\begin{abstract}
Microgel and macrogel formation during the photodimerization of cinnamoyl groups in polymer chains has been investigated through analysis of intramolecular crosslinks using spectrophotometric and light scattering measurements. The form of gels depended particularly on the amount of cinnamoyloxyethyl methacrylate (CEMA) in copoly (CEMA/MMA). For CEMA= $12 \mathrm{~mol} \%$ copolymer, microgels were formed in the early stage of reaction, and finally homogeneous macrogels in appearance were formed. The resulting microgel for CEMA $=26 \mathrm{~mol} \%$ copolymer was composed of networks having the number of intramolecular crosslinked cinnamoyl groups, $n_{\text {intra }}=$ 1870 in 3400 units of cinnamoyl groups per polymer chain. At the onset of gelation, the volume of polymer coils due to intramolecular crosslinks became $1 / 350-1 / 10$ the volume of the corresponding linear polymer of the same molecular weight. The extent of intra- and intermolecular crosslinks and contraction of polymer coils due to intramolecular crosslinks were estimated in the process of gel formation.
\end{abstract}

KEY WORDS Microgel / Macrogel / Intramolecular Crosslink / Photocrosslinking / Photodimerization / Cinnamoyl Group / Contraction of Polymer Coil / Light Scattering / Onset of Gelation /

The formation of a three-dimensional network of polymer molecules has been investigated by many authors. ${ }^{1-10}$ When polymer molecules are crosslinked in solution, either during polymerization or by polymer reactions, both intra- and intermolecular crosslinks can be formed. In the radical copolymerization of styrene and divinylbenzene in solution, the extent of competition between intra- and intermolecular crosslinking and intramolecular cyclization between adjacent units has been discussed. ${ }^{2.3 .5}$ White et al. ${ }^{2}$ have reportd that the probability of intramolecular cyclization is rather high, whereby the number of elastically effective network chains is reduced. Horie et $a l{ }^{3}$ have also revealed three kinds of modes for crosslinking from an experimental study on the crosslinked copolymerization of methylmethacrylate (MMA) with ethylene dimethacrylate (EDMA) in bulk and in toluene. It had been shown that crosslinked copolymerization depends on the initial composition of monomers and concentration in toluene, and intramolecular crosslinking between nonadjacent units and the formation of microgels are important characteristics for copolymerization with higher divinyl contents. Matsumoto $e t$ $a l^{4,9}$ have examined the gelation for the polymerization of diallyl esters of aromatic dicarboxylic acids and discussed the results according to Gordon's theory. ${ }^{1}$ They concluded that the intramolecular chain transfer reaction plays an important role during polymerization. The gel formation due to photogener- 
ated crosslinks in solid state has been investigated. ${ }^{11-13}$ The intramolecular crosslinking is negligible for irradiation of solid poly(vinyl cinnamate), and mainly intermolecular crosslinks are formed. ${ }^{12}$

We recently reported kinetic parameters during the simultaneous occurrence of intraand intermolecular photocrosslinking in polymers containing cinnamoyl groups in dilute solution. ${ }^{14-16}$ The quantum yields for intramolecular crosslink formation increase linearly with increasing cinnamoyl groups in a polymer. The parameters of intermolecular crosslink formation, in contrast, decrease with the extent of the reaction, because the increase in intramolecular crosslinks prevents interpenetration of polymer coils. In the present paper, microgel and macrogel formation during the photodimerization of cinnamoyl groups in polymer chains was studied by analysis of the intramolecular crosslinks. Several methyl methacrylate (MMA) copolymers with 2-cinnamoyloxyethyl methacrylate (CEMA) were irradiated in aerated and degassed tetrahydrofuran solutions under $\mathrm{Hg}$ lamp at $30^{\circ} \mathrm{C}$. The degree of conversion due to cyclodimer formation of the cinnamoyl groups in solution was measured from the change in the UV absorption band with a maximum at $275 \mathrm{~nm}$. The extent of intermolecular crosslinking was estimated from the change in molecular weight measured by light scattering. The extent of intra- and intermolecular crosslinks was estimated in the process of microgel and macrogel formation, and consequently, the microgel and macrogel formed contain many intramolecular crosslinks. The magnitude of the contraction of polymer coils due to intramolecular crosslinking is also briefly discussed.

\section{EXPERIMENTAL}

Copolymers were prepared as reported previously. ${ }^{16}$ The copolymerization of 2-hydroxyethyl methacrylate (HEMA) and methyl methacrylate (MMA) was carried out in dimethylformamide as the solvent under a vacuum at $60^{\circ} \mathrm{C}$. The copoly(HEMA/MMA) samples were reacted with cinnamoyl chloride, and the resulting copolymers with cinnamoyl groups were further fractionated by dissolving them in a mixed solvent of benzene and methanol and changing stepwise the temperature of the solution from 50 to $20^{\circ} \mathrm{C}$. The characteristics of the copolymers are given in Table I.

Sample solutions $\left(3.4-0.2 \mathrm{gl}^{-1}\right)$ in a pyrex cell were deaerated by several freeze-pumpthaw cycles at $10^{-4}$ Torr, and sealed off under high vacuum. For an aerated sample, the sample solutions were sealed off without evacuation. The sample solutions were irradiated with light of $300-410 \mathrm{~nm}$ from a $450 \mathrm{~W}$ highpressure $\mathrm{Hg}$ lamp (Ushio UM-452) with a glass filter of Toshiba UV-D33S in a thermostat at $30^{\circ} \mathrm{C}$. To minimize the influence of the heterogeneous nature of the reaction, the solution in the cell was continuously stirred magnetically during irradiation. Actinometry was carried out with an Ushio UIT-100 Type photoresist photometer calibrated by the potassium ferrioxalate/o-phenanthroline system. ${ }^{17}$ The incident light intensity was about $I_{0}=1.26 \times 10^{-8}$ einstein $\mathrm{cm}^{-2} \mathrm{~s}^{-1}$.

The content of cinnamoyl groups in the

Table I. Characterization of polymers containing cinnamoyl groups

\begin{tabular}{|c|c|c|c|c|c|c|}
\hline \multirow{2}{*}{ Polymer } & \multirow{2}{*}{$\frac{\mathrm{UV}}{[\mathrm{C}] / \mathrm{mol}^{\%} /}$} & \multirow{2}{*}{$\frac{\mathrm{GPC} / \mathrm{THF}}{\bar{M}_{w} / \bar{M}_{n}}$} & \multicolumn{3}{|c|}{ Light-scattering } & \multirow{2}{*}{$\bar{N}_{n}$} \\
\hline & & & $\bar{M}_{w} / 10^{6}$ & $\left\langle S^{2}\right\rangle^{1 / 2} / 10^{2} \AA$ & $A_{2} / 10^{-4} \mathrm{~mol} \mathrm{ml} \mathrm{g}^{-2}$ & \\
\hline $6400-2.4$ & 2.40 & 2.0 & 1.35 & 7.4 & 1.1 & 6400 \\
\hline $5100-12$ & 11.7 & 1.8 & 1.09 & 5.1 & 0.86 & 5100 \\
\hline $5600-26$ & 26.0 & 2.3 & 1.85 & 5.3 & 0.97 & 5600 \\
\hline
\end{tabular}


copolymers and extent of cyclodimer formation of the cinnamoyl groups in solution were evaluated from the change in optical density at the maximum wavelength $(275 \mathrm{~nm})$ as reported previously. ${ }^{18}$ The weight-average molecular weight, $\bar{M}_{w}$, root-mean-square radius of gyration, $\left\langle s^{2}\right\rangle^{1 / 2}$, and the second virial coefficient, $A_{2}$, were measured by a Union Giken LS-601 Type light scattering photometer, and the molecular weight distribution of the polymers was determined with a Toyo Soda HLC-802UR gel permeation chromatograph at $40^{\circ} \mathrm{C}$ in tetrahydrofuran (THF). The details of this determination have already been given. ${ }^{15}$

\section{RESULTS AND DISCUSSION}

The change in concentration of cinnamoyl groups of copoly(CEMA/MMA) due to cyclodimer formation in degassed THF was evaluated from changes in the UV absorption spectra as reported previously. ${ }^{14}$ The results for $\mathrm{CEMA}=12 \mathrm{~mol} \%$ copolymer are shown Figure 1, and the conversion, $x=1-[\mathrm{C}] /[\mathrm{C}]_{0}$, of photodimerization of cinnamoyl groups was calculated from plots of residual concentration against irradiation time, $t$, For competitive

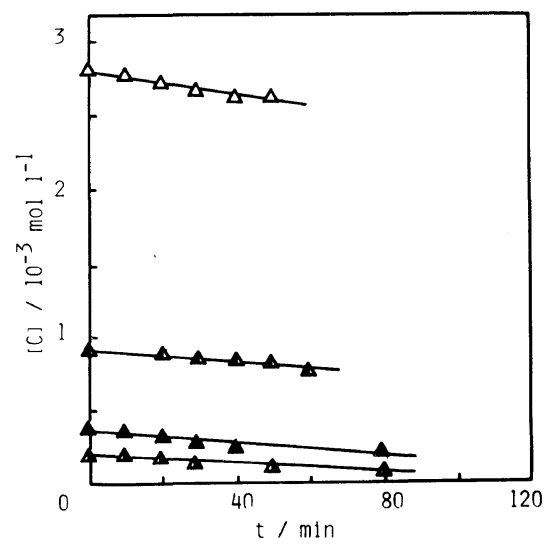

Figure 1. Change in the concentration of cinnamoyl groups of a copolymer (5100-12) due to photocrosslinking in degassed $\mathrm{THF}$ at $30^{\circ} \mathrm{C}$. Concentration $/ \mathrm{g}^{-1}$ $\left(\mathrm{mol} \mathrm{l}^{-1}\right): \triangle, 3.4\left(2.8 \times 10^{-3}\right) ; \Delta, 1.1\left(0.95 \times 10^{-3}\right) ; \mathbf{\Delta}$ $0.48\left(0.40 \times 10^{-3}\right) ; \boldsymbol{\Delta}, 0.27\left(0.23 \times 10^{-3}\right)$. intra- and intermolecular crosslink formation, the extent of intermolecular crosslinking was estimated from the change in molecular weight measured by light scattering. Figure 2 shows the plots of $\bar{M}_{w}$ against the conversion, $x$, for photocrosslinking of CEMA $=12 \mathrm{~mol} \%$ copolymer. The change in $\bar{M}_{w}$ becomes very marked with increasing initial polymer concentration, and at $x=0.07$ for the experiment with $[C]_{0}=3.4 \mathrm{~g} \mathrm{l}^{-1}$, the value of $\bar{M}_{w}$ is about 20 times that of the initial molecular weight. However, at $[\mathrm{C}]_{0}=0.48 \mathrm{~g} \mathrm{I}^{-1}$, the increase in $\bar{M}_{w}$ is depressed by competitive intramolecular crosslinking.

The number of intermolecular crosslinks per polymer chain, $\rho_{\mathrm{c}} \bar{N}_{w(\mathrm{c}, 0)}$, is expressed ${ }^{19}$ :

$$
\rho_{\mathrm{c}} \bar{N}_{w(\mathfrak{c}, 0)}=1-\frac{\bar{M}_{w(0)}}{\bar{M}_{w}}
$$

were $\rho_{\mathrm{c}}$ is crosslinking density based on the number of chromophore units in the copolymer, and $\bar{N}_{w(\mathrm{c}, 0)}$ and $\bar{M}_{w(0)}$ are the weightaverage number of CEMA units per copolymer chain and the weight-average molecular weight of the copolymer before crosslinking, respectively. Figures $3-5$ show plots of $\rho_{\mathrm{c}} \bar{N}_{w(\mathrm{c} .0)}$ against both conversion of the cin-

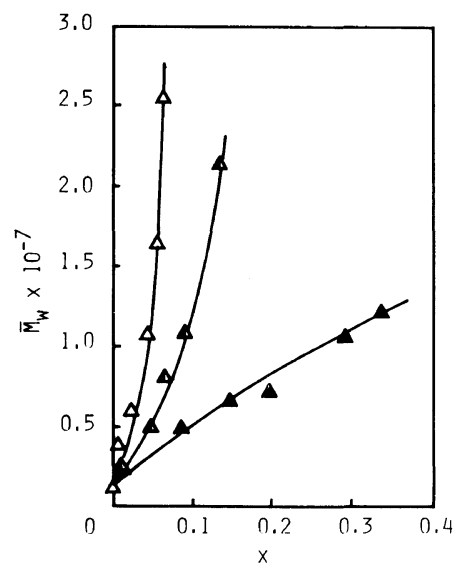

Figure 2. Variation in weight-average molecular weight against the conversion of cinnamoyl groups for photocrosslinking of a copolymer $(5100-12)$ in degassed THF at $30^{\circ} \mathrm{C}$. Concentration $/ \mathrm{g}^{-1}: \triangle, 3.4$; $\triangle, 1.1 ; \Delta, 0.48$. 


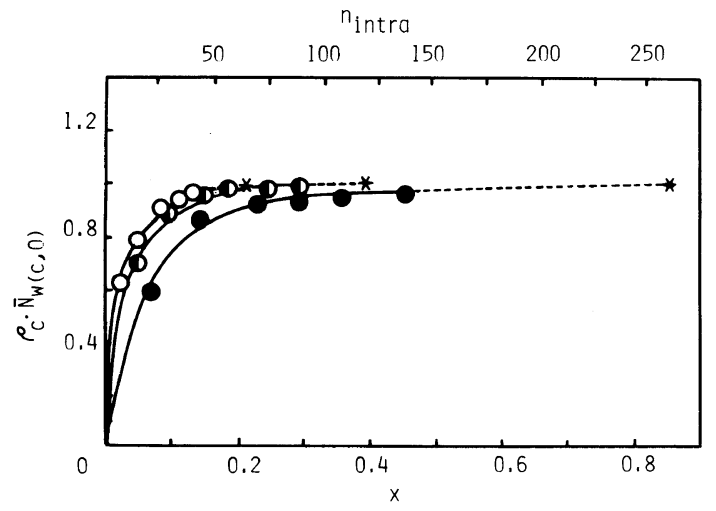

Figure 3. Plots of the rate of intermolecular crosslinks per polymer chain against the conversion of cinnamoyl groups and the number of cinnamoyl groups for intramolecular crosslinks per chain for irradiation of a copolymer $(6400-2.4)$. Concentration $/ \mathrm{g}^{-1} ; \bigcirc, 3.4 ; \boldsymbol{D}$, $1.1 ; 0.47 ; *$, the onset of gelation.

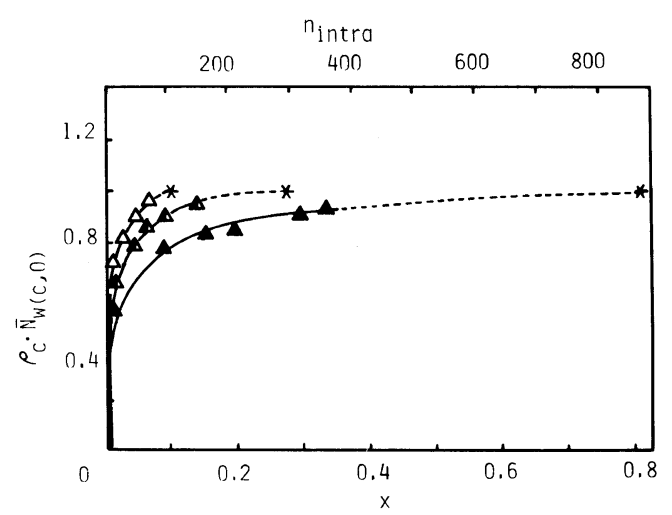

Figure 4. Plots of the rate of intermolecular crosslinks per polymer chain against the conversion of cinnamoyl groups and the number of cinnamoyl groups for intramolecular crosslinks per chain for irradiation of a copolymer $(5100-12)$. Concentration $/ \mathrm{g}^{-1}: \triangle, 3.4 ; \triangle$, 1.1; $\mathbf{\Delta}, 0.48 ; *$, the onset of gelation.

namoyl groups, $x$, and the number of cinnamoyl groups used for intramolecular crosslinks per polymer chain, $n_{\text {intra }}$. The value of $n_{\text {intra }}$ was evaluated by subtracting the value of $\rho_{\mathrm{c}} \bar{N}_{w(\mathrm{c}, 0)}$ from the number of dimerized cinnamoyl groups per polymer chain. The star symbol represents the point of the theoretical onset of gelation at $\rho_{\mathrm{g}}=1 / \bar{N}_{w(\mathrm{c}, 0)}$ for $\bar{M}_{w} \gg \bar{M}_{w(0)}$. The values of $\rho_{\mathrm{c}} \bar{N}_{w(\mathrm{c}, 0)}$ increase rapidly in the early stage of crosslinks for-

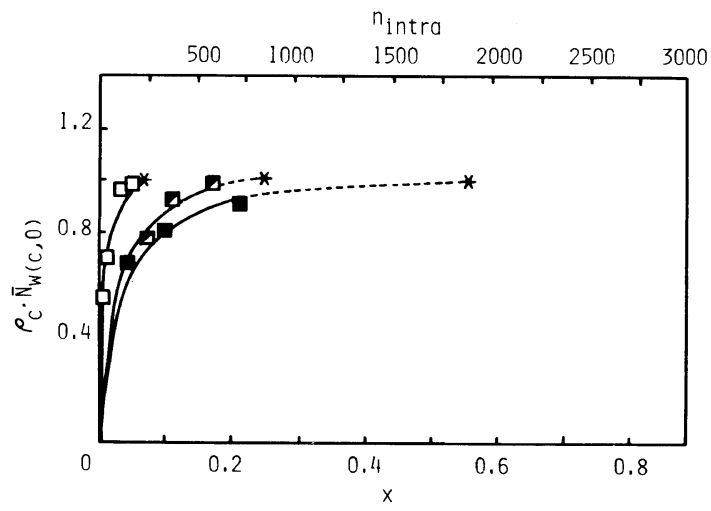

Figure 5. Plots of the rate of intermolecular crosslinks per polymer chain against the conversion of cinnamoyl groups and the number of cinnamoyl groups for intramolecular crosslinks per chain for irradiation of a copolymer $(5600-26)$. Concentration $/ \mathrm{g}^{-1}: \square, 3.4 ; \square$, $1.1 ; \square, 0.47 ; *$, the onset of gelation.

mation, becoming close to $\rho_{\mathrm{c}} \bar{N}_{w(\mathrm{c}, 0)}=1$ in all cases. The abrupt increases in $\rho_{\mathrm{c}} \bar{N}_{w(\mathrm{c}, 0)}$ becomes greater as the initial polymer concentration increases. For the experiment with $[\mathrm{C}]_{0}=3.4 \mathrm{gl}^{-1}$ of $\mathrm{CEMA}=2.4 \mathrm{~mol} \%$ copolymer as shown in Figure 3, the network formation of macrogel was observed for $x=$ $0.20-0.23$. This value corresponds to $60-70$ cinnamoyl units in 307 units of initial cinnamoyl groups per copolymer chain. Because of the restriction of the conformational changes in polymer coils due to intramolecular crosslinks, the value of $\rho_{\mathrm{c}} \bar{N}_{w(\mathrm{c}, 0)}$ deviates from linearity during the crosslinking. This tendency becomes more pronounced as the initial polymer concentration decreases. However, the macrogels formed were colorless and very viscous, regardless of the initial polymer concentration of the solutions.

For $[C]_{0}=3.4-0.48 \mathrm{gl}^{-1}$ of $\mathrm{CEMA}=12$ $\mathrm{mol} \%$ copolymer as shown in Figure 4 , the formation of a slightly white microgel was occasionally observed near $\rho_{\mathrm{c}} \bar{N}_{w(\mathrm{c}, 0)}=1$, but the white color disappeared again as crosslinking proceeded. This means that in the early stage of crosslinking, the predominant intramolecular crosslinking results in the formation of microgels with a heterogeneity in the density 
of the solution, but as the reaction proceeds, these microgels approach close to each other and are crosslinked intermolecularly by reactions between cinnamoyl groups at the surface of different microgels, and the microgels are transformed to macrogels which are transparent and homogeneous in appearance. At $[C]_{0}=3.4 \mathrm{~g} \mathrm{l}^{-1}$, the resulting macrogel is composed of networks having $n_{\text {intra }}=110$ in 1074 units of cinnamoyl groups per polymer chain. For the experiment with $[\mathrm{C}]_{0}=0.48 \mathrm{gl}^{-1}$, the value of $n_{\text {intra }}$ is 870 at the point of gelation and the product is a highly intramolecularcrosslinked macrogel.

For the $\mathrm{CEMA}=26 \mathrm{~mol}^{\circ}$ copolymer as shown in Figure 5 , at $[C]_{0}=0.47 \mathrm{~g}^{-1}$, the

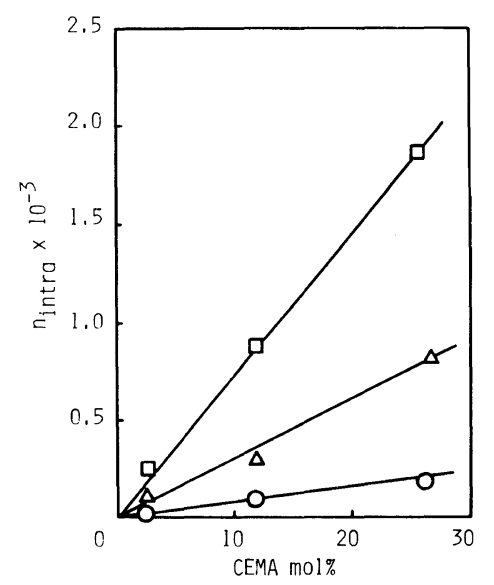

Figure 6. Dependence of the number of cinnamoyl groups for intramolecular crosslinks per chain on CEMA $\mathrm{mol} \%$ in copolymers at the onset of gelation. Concentration $/ \mathrm{gl}^{-1}: 0,3.4 ; \triangle, 1.1 ; \square, 0.47-0.48$. microgel formed is composed of networks having $n_{\text {intra }}=1870$ in 3400 units of cinnamoyl groups per polymer chain. As the crosslinking proceeds further, these microgels are not transformed to macrogels and the crosslinking reaction inside of microgels takes place rather preferentially, leading to densely packed and heterogeneous discrete microgel particles. This phenomenon was also observed for the experiments with $[\mathrm{C}]_{0}=1.1$ and $3.4 \mathrm{gl}^{-1}$.

The number of cinnamoyl groups for intramolecular crosslinks per polymer chain at the onset of gelation, $n_{\text {intra }}$, is plotted in Figure 6 as a function of the cinnamoyl content in copolymers. The results are listed in Table II.

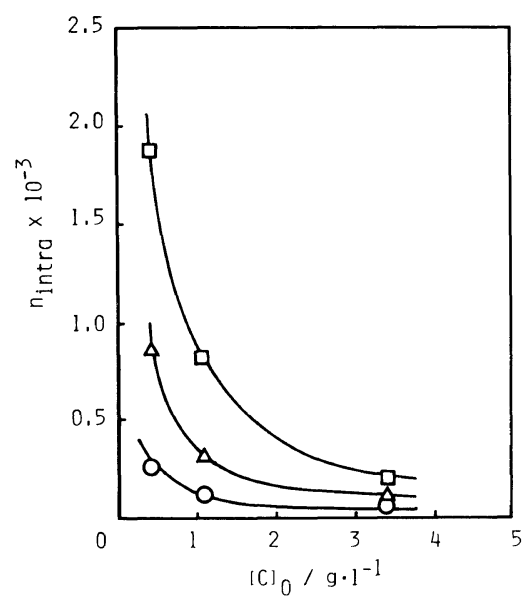

Figure 7. Dependence of the number of cinnamoyl groups for intramolecular crosslinks per chain on the initial concentration of copolymers at the onset of gelation. Copolymers: $\bigcirc, 6400-2.4 ; \triangle, 5100-12 ; \square$, $5600-26$.

Table II. Effect of CEMA mol $\%$ in copoly(CEMA/MMA) on the onset of gelation due to photocrosslinking reaction in solution at $30^{\circ} \mathrm{C}$

\begin{tabular}{|c|c|c|c|c|c|c|c|c|c|c|c|}
\hline \multirow{2}{*}{ Polymer } & \multirow{2}{*}{$\begin{array}{l}\text { CEMA } \\
\mathrm{mol}^{\circ} \%\end{array}$} & \multicolumn{3}{|c|}{$n_{\text {intra }}(x)$} & \multicolumn{3}{|c|}{$g$ value } & \multicolumn{3}{|c|}{$A_{2} / 10^{-5} \mathrm{~mol} \mathrm{ml} \mathrm{g}^{-2}$} & \multirow{2}{*}{$\begin{array}{l}\text { Network } \\
\text { formation }\end{array}$} \\
\hline & & $0.47(\mathrm{~g} / \mathrm{l})$ & 1.1 & 3.4 & 0.47 & 1.1 & 3.4 & 0.47 & 1.1 & 3.4 & \\
\hline $6400-2.4$ & 2.4 & $\begin{array}{c}260 \\
(0.84)\end{array}$ & $\begin{array}{c}120 \\
(0.39)\end{array}$ & $\begin{array}{c}70 \\
(0.23)\end{array}$ & 0.02 & 0.1 & 0.3 & 0.1 & 0.2 & 0.7 & Macrogel \\
\hline $5100--12$ & 11.7 & $\begin{array}{c}870 \\
(0.81)\end{array}$ & $\begin{array}{c}300 \\
(0.28)\end{array}$ & $\begin{array}{c}110 \\
(0.10)\end{array}$ & 0.2 & 0.2 & 0.3 & 0.2 & 0.3 & 1.8 & Macrogel \\
\hline $5600-26$ & 26.0 & $\begin{array}{c}1870 \\
(0.55)\end{array}$ & $\begin{array}{c}815 \\
(0.24)\end{array}$ & $\begin{array}{c}200 \\
(0.06)\end{array}$ & 0.02 & 0.1 & 0.3 & 0.2 & 0.1 & 0.2 & Microgel \\
\hline
\end{tabular}


The values of $n_{\text {intra }}$ increase linearly with cinnamoyl content in copolymers for various initial polymer concentration. The cinnamoyl groups in a copolymer are effectively used for the network formation of microgels or macrogels in solution. The gel formed contains many intramolecular crosslinks. Figure 7 shows plots of $n_{\text {intra }}$ against the initial polymer concentration. The values of $n_{\text {intra }}$ increase rapidly below $[\mathrm{C}]_{0}=1 \mathrm{gl}^{-1}$, and the abrupt increase of $n_{\text {intra }}$ becomes greater with a the content of cinnamoyl groups in a copolymer.

From the light-scattering measurements, we could obtain the relationship of second virial coefficient, $A_{2}$, with $\bar{M}_{w}$ as shown in Figure 8. The second virial coefficient becomes progressively smaller as crosslinking proceeds, and reaches almost zero at the onset of gelation. Because of the intramolecular crosslinking, the rate of decrease in $A_{2}$ against $\bar{M}_{w}$ becomes greater with increasing CEMA mol $\%$ in a copolymer. For CEMA $=12 \mathrm{~mol} \%$ copolymer as shown in Table II the value of $A_{2}$ at the onset of gelation becomes greater as the initial polymer concentration increases, compared with others. This may be attributed to the formation of macrogels.

The values of root-mean-square radius, $\left\langle s^{2}\right\rangle^{1 / 2}$, were plotted on a double logarithmic scale against $\bar{M}_{w}$ for $[C]_{0}=0.47-0.48 \mathrm{~g} \mathrm{l}^{-1}$ in Figure 9. For the CEMA $=26 \mathrm{~mol}^{\circ} \%$ copoly-

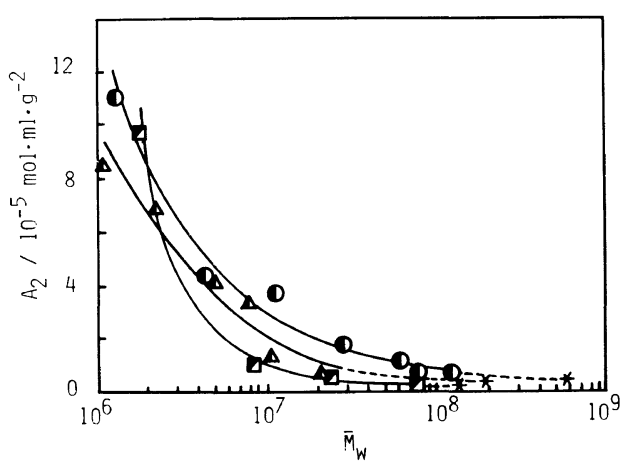

Figure 8. Plots of second virial coefficient against weight-average molecular weight for irradiated copolymers at $[\mathrm{C}]_{0}=1.1 \mathrm{~g} \mathrm{l}^{-1}$. Copolymers: $\mathrm{D}, 6400-2.4 ; \Delta$, $5100-12 ; \square, 5600-26 ; *$, the onset of gelation. mer which form microgels as the crosslinking proceeds, the dimension of polymer coils decreases despite the large value of $\bar{M}_{w}$. This behavior is attributed to the increase of the contraction caused by intramolecular crosslinking overcoming the increase of expansion caused by intermolecular crosslinking. To evaluate the net contraction of polymer chains due to intramolecular crosslinking, the contraction ratio, $g$, is given by eq 2 as reported previously, ${ }^{15}$

$$
g=\left\langle s^{2}\right\rangle_{\mathrm{cr}} /\left\langle s^{2}\right\rangle_{1}
$$

The values of $\left\langle s^{2}\right\rangle_{\text {cr }}$ are those of $\left\langle s^{2}\right\rangle$ for irradiated polymers given in Figure 9. The values of $\left\langle s^{2}\right\rangle_{1}$ were calculated with the value

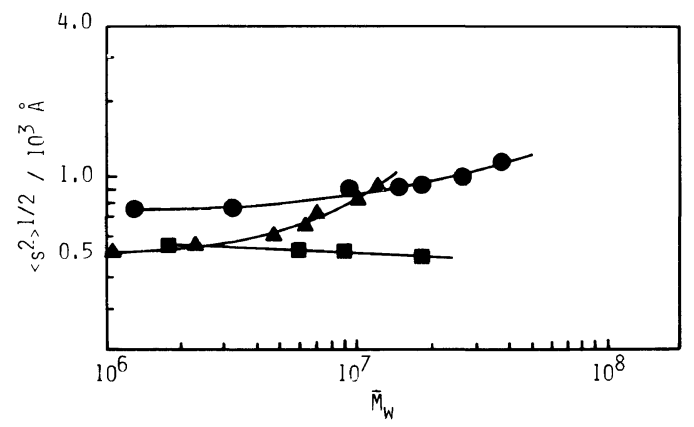

Figure 9. Molecular-weight dependence of root-meansquare radius of gyration for irradiation of copolymers at $[C]_{0}=0.47-0.48 \mathrm{~g}^{-1}$. Copolymers: O, $6400-2.4$; A, $5100-12 ; \boldsymbol{\square}, 5600-26$.

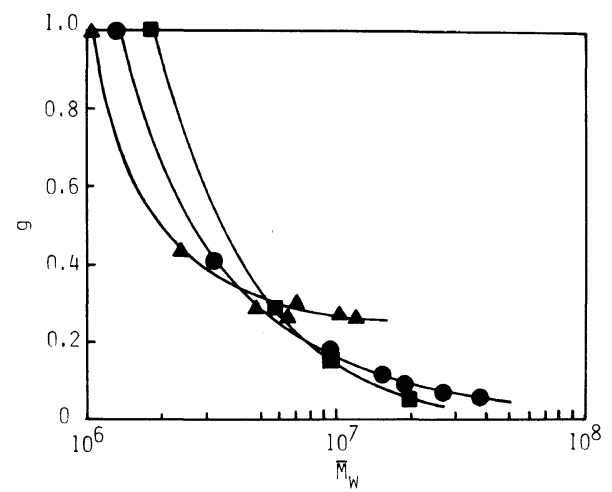

Figure 10. Plots of $g$ against weight-average molecular weight for copolymers at $[\mathrm{C}]_{0}=0.47-0.48 \mathrm{gl}^{-1}$. Copolymers: $\bigcirc, 6400-2.4 ; \boldsymbol{\Lambda}, 5100-12 ; \mathbf{\square}, 5600-26$. 


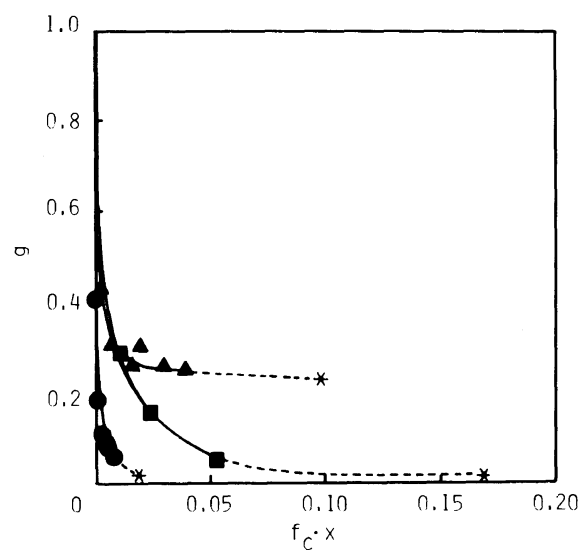

Figure 11. Plots of $g$ against $f_{\mathrm{c}} x$ for copolymers at $[C]_{0}=0.47-0.48 \mathrm{~g}^{-1}$. Copolymers:, $6400-2.4 ; \boldsymbol{\Delta}$, $5100-12 ; \square, 5600-26 ; *$, the onset of gelation.

of $\left\langle s^{2}\right\rangle_{1,0}$ before irradiation and the molecular weight using the relationship $\left\langle s^{2}\right\rangle_{1} \propto$ $M^{(2 / 3)(1+\alpha)}$ where $a$ is the viscosity coefficient, assumed to be 0.6 . The contraction ratio, $g$, plotted against $\bar{M}_{w}$ decreases with the progress of reaction, more rapidly at the initial stage of reactions for experiments with $[\mathrm{C}]_{0}=$ $0.47-0.48 \mathrm{~g}^{-1}$ as shown in Figure 10. Figure 11 shows the change of the value of $g$ against the extent of dimerization of cinnamoyl groups, $f_{\mathrm{c}} x$, where the fraction, $f_{\mathrm{c}} x$, of dimerized chromophore units is defined as the conversion, $x$, multiplied by a cinnamoyl fraction in the copolymer composition. The values of $g$ become $0.02-0.2$ at the onset of gelation. The value of $g$ for each copolymer is listed in Table II. This value means that the volume of the crosslinked polymer coil shrinks to $1 / 350$ $1 / 10$ the volume of the corresponding linear polymer having the same molecular weight.

In conclusion, the effect of intramolecular crosslinks on the microgel and macrogel formation in solution was elucidated by analysis of the photodimerization of copolymers having cinnamoyl groups. The formation of gels during the competitive intra- and intermolecular photocrosslinking in solution depends on the amount of CEMA in copoly(CEMA/ MMA) and the initial polymer concentra- tion. For the CEMA $=12 \mathrm{~mol} \%$ copolymer, microgels are formed in the early stage of reaction and are connected with one another in the later stage, and finally homogeneous macrogels in appearance are formed. The number of intramolecular crosslinks per chain at the onset of gelation increases rapidly for $[\mathrm{C}]_{0}<1 \mathrm{~g} \mathrm{l}^{-1}$. The resulting gels consist of many intramolecular crosslinks in all cases. The contraction of polymer coils due to intramolecular crosslinks contributes to the restriction of the segmental mobility of polymer coils and to decrease in chain interpenetration.

\section{REFERENCES}

1. M. Gordon, J. Chem. Phys., 22, 610 (1954).

2. B. Soper, R. N. Harward, and E. F. T. White, J. Polym. Sci., A-1, 2545 (1972).

3. K. Horie, A. Otagawa, M. Muraoka, and I. Mita, $J$. Polym. Sci., Polym. Chem. Ed., 13, 445 (1975).

4. A. Matsumoto, S. Yokoyama, T. Khono, and M. Oiwa, J. Polym. Sci., Phys. Ed., 15, 127 (1977).

5. K. Dusek, M. Gordon, and S. B. Ross-Murphy, Macromolecules, 11, 236 (1978).

6. K. Dusek and J. Spevacek, Polymer, 21, 750 (1980).

7. H. Galina, K. Dusek, Z. Tuzar, M. Bohdanecky, and J. Stokr, Eur. Polym. J., 16, 1043 (1980).

8. J. Spevacek and K. Dusek, J. Polym. Sci., Polym. Phys. Ed., 18, 2027 (1980).

9. A. Matsumoto, Y. Naito, and M. Oiwa, Polym. Prepr. Jpn., 33, 1347 (1984).

10. I. Mita and K. Horie, J. Macromol. Sci., Rev. Macromol. Chem. Phys., C27, 91 (1987).

11. A. Reiser and P. L. Egerton, Macromolecules, 12, 670 (1979).

12. P. L. Egerton, E. Pitts, and A. Reiser, Macromolecules, 14, 95 (1981).

13. A. Matsumoto, A. Fukazawa, and M. Oiwa, $J$. Appl. Polym. Sci., 28, 11 (1983).

14. Y. Shindo, T. Sugimura, K. Horie, and I. Mita, Nippon Kagaku Kaishi, 184 (1984).

15. Y. Shindo, T. Sugimura, K. Horie, and I. Mita, Eur. Polym. J., 22, 859 (1986).

16. Y. Shindo, H. Sato, T. Sugimura, K. Horie, and I. Mita, Eur. Polym. J., in press.

17. S. L. Murov. "Handbook of Photochemistry," Dekker, New York, N.Y., 1973, p 119.

18. Y. Shindo, K. Horie, and I. Mita, Chem. Lett., 639 (1983).

19. C. David, D. Baeyens-Volant, Eur. Polym. J., 14, 29 (1978). 